\title{
Endoscopic sleeve gastroplasty: a modified technique with greater curvature compression sutures
}

\section{다(1) $(9$}

\author{
Authors \\ Michael A. Glaysher, Alma L. Moekotte, Jamie Kelly \\ Institution \\ Department of Upper Gastrointestinal Surgery, University \\ Hospital Southampton NHS Foundation Trust, Tremona \\ Road, Southampton, Hampshire, UK
}

submitted 19.3.2019

accepted after revision $\quad 3.6 .2019$

Bibliography

DOI https://doi.org/10.1055/a-0996-8089 |

Endoscopy International Open 2019; 07: E1303-E1309

(c) Georg Thieme Verlag KG Stuttgart · New York

eISSN 2196-9736

Corresponding author

Michael A. Glaysher, BM (Hons), MRCS Eng, Department of Upper Gastrointestinal Surgery, University Hospital

Southampton NHS Foundation Trust, Tremona Road,

Southampton, Hampshire SO16 6YD, UK

michaelglaysher@me.com

\section{ABSTRACT}

Background Endoscopic sleeve gastroplasty (ESG) is rapidly becoming established as a safe and effective means of achieving substantial weight loss via the transoral route. New ESG suture patterns are emerging. Our aim was to investigate whether superior weight loss outcomes can be achieved by using a unique combination of longitudinal compression sutures and "U"-shaped sutures.
Methods This is a retrospective review of prospectively collected data of all patients undergoing ESG by a single operator in a single UK center.

Results Between January 2016 and December 2017, 32 patients ( 23 female) underwent ESG; $n=9$ cases were completed utilizing a commonly used triangular suture pattern ("no longitudinal compression") and $\mathrm{n}=23$ cases were completed using our unique "longitudinal compression" suture pattern. In the no compression and compression groups, the mean ages were $45 \pm 12$ years and $43 \pm 10$ years, the median baseline weights were $113.6 \mathrm{~kg}$ (range $82.0-156.4$ ) and $107 \mathrm{~kg}$ (range $74.0-136.0$ ), and the median baseline body mass indexes (BMIs) were $35.9 \mathrm{~kg} / \mathrm{m}^{2}$ (range $30.9-$ 43.8 ) and $36.5 \mathrm{~kg} / \mathrm{m}^{2}$ (range $29.8-42.9$ ), respectively. After 6 months, body weight had decreased by $21.1 \mathrm{~kg}$ (range, $12.2-34.0)$ in the compression group $(n=7)$ versus $10.8 \mathrm{~kg}$ (range, $7.0-25.8$ ) in the no compression group $(\mathrm{n}=$ 5) $(P=0.042)$. Correspondingly, BMI decreased by $7.8 \mathrm{~kg} / \mathrm{m}^{2}$ (range, $4.9-11.2$ ) and $4.1 \mathrm{~kg} / \mathrm{m}^{2}$ (range, $2.6-7.2$ ) in each group, respectively $(P=0.019)$. Total body weight loss $(\%$ TBWL) was greater in the compression group at $19.5 \%$ (range, $12.9-30.4 \%$ ) compared to $13.2 \%$ (range, 6.2 $17.1 \%)$ in the non-compression group $(P=0.042)$. No significant adverse events were reported in this series.

Conclusion The technique of ESG is evolving and outcomes from endoscopic bariatric therapies continue to improve. We provide preliminary evidence of superior weight loss achieved through a modified gastroplasty suture pattern.

\section{Background}

Obesity and obesity-related diseases are preventable conditions that represent a significant socioeconomic burden. In 2017, the World Health Organization (WHO) estimated that $13 \%$ of the world's adult population were obese (clinically defined as a body mass index (BMI) of $\geq 30 \mathrm{~kg} / \mathrm{m}^{2}$ ) and this incidence has tripled since 1975 [1]. In the UK alone, it has been projected that there will be an additional 11 million obese adults by 2030. Metabolic surgery remains the most effective long-term means of treating these patients by producing often profound and sustained weight loss, as well as weight-loss independent improvements in metabolic health, which consequently ameliorate, or even eliminate, associated comorbidities and reduce mortality [ $2-4]$. The safety profile of metabolic surgery has improved markedly in recent years with quoted mortality rates of $0.1-0.5 \%$ worldwide $(0.11 \%$ in the UK) but with serious morbidity recorded in up to $6 \%$ of patients [5, 6$]$. Unfortunately, only a small proportion of eligible, obese patients (approximately $1 \%$ ) currently undergo metabolic operations as there continues to be numerous, multifactorial barriers to surgery [7]. 
Endoscopic bariatric therapies (EBTs) provide a minimally invasive, and potentially cost-effective, therapeutic option to achieve weight-loss and treat obesity-related diseases by going beyond what can be achieved through medical and lifestyle interventions alone while limiting the potential morbidity and mortality associated with surgery.

First described in 2008, endoscopic sleeve gastroplasty (ESG) is rapidly becoming established as an effective means of achieving significant weight loss via the transoral route [8]. The generally accepted technique of ESG uses the OverStitch (Apollo Endosurgery Inc., Austin, TX, United States), cap-based flexible endoscopic suturing system which is mounted onto a double-channel endoscope. Starting distally at the level of the incisura angularis and working proximally along the greater curvature towards the fundus, the OverStitch device is used to fashion multiple, interrupted triangular plications. This creates concentric compression along the greater curvature that results in the formation of a short tubular gastric lumen and a $70 \%$ reduction in stomach volume without the need to amputate the greater curvature, as is required in laparoscopic sleeve gastrectomy. Weight-loss is achieved by significantly restricting the volume of food consumed at each meal coupled with a delay in gastric emptying, which promotes early satiety [9]. This now widely accepted technique, as described by Lopez-Nava et al. [10], has excellent short-term safety and efficacy outcomes reported in numerous prospective observational studies [925]. After 2 years, patients can expect to achieve a total body weight loss (\%TBWL) of $15-20 \%$ and two studies have reported associated improvements in several metabolic parameters [9, $13,26]$. Following ESG, patients report only mild-to-moderate, transient, postoperative symptoms and significant adverse events have been reported in selected studies at a rate of $<2 \%$ $[9,11,13,14]$.

Multiple ESG suture patterns already exist that are based broadly around a triangular configuration of plications whereby tension on these plications acts to reduce the volume of the gastric cavity by concentrically compressing the greater curvature with some additional shortening of the sleeve ( $\mathbf{F i g . 1}$ ). More recently, several different " $Z$ "-shaped running suture patterns have also been trialled $[12,14]$. Graus Morales et al. (2018) demonstrated comparable short-term results using this technique but failed to demonstrate superiority of this suture pattern over the "conventional" triangular pattern in terms of weight-loss outcomes after 18 months.

In our center, a modified suture pattern was designed in order to optimize the biomechanical compressive forces acting along the greater curvature. Through the addition of longitudinal compression sutures, tension is more equally distributed across each stitch and produces maximal, uniform compression in both anterior-posterior (A-P) and craniocaudal dimensions. With better, more homogenous compression and greater volume reduction in this manner, we hypothesize that this will translate to earlier satiety and superior weight loss outcomes.

Herein, we report the 6-month outcomes of consecutive patients undergoing ESG by a single operator in our center, comparing the previously described triangular configuration of plications ( $\triangleright$ Fig. 1 ) against our modified suture configuration

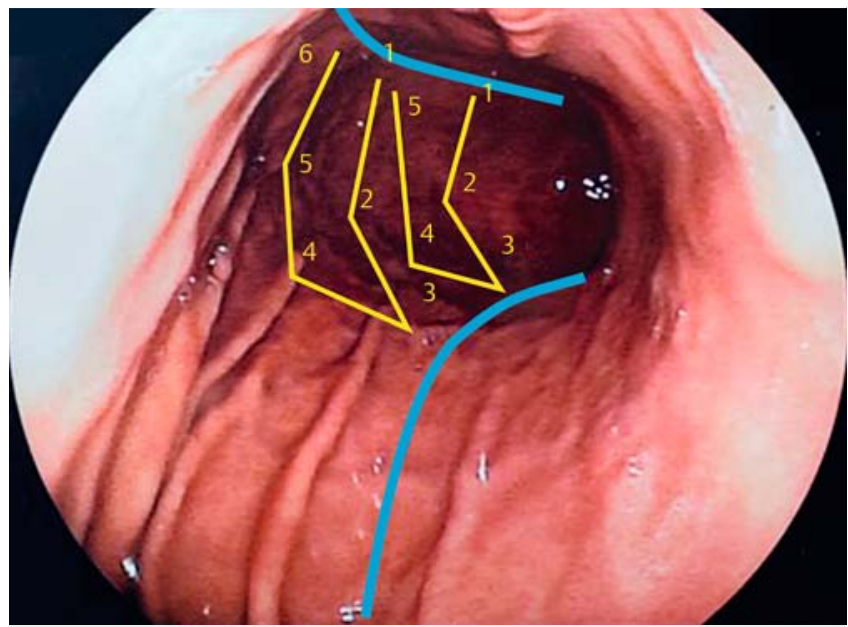

- Fig. 1 Schematic representation of the conventional triangular suture pattern used by Lopez-Nava et al. [10] (no longitudinal compression).

that incorporates longitudinal compression sutures along the greater curvature ( $\triangleright$ Fig. 2 ).

\section{Patients and methods}

\section{Patients}

This is a retrospective review of prospectively collected data of all patients undergoing ESG by the senior author (JK) in a single center (Spire Healthcare, Southampton, UK) between January 2016 and December 2017. JK is established as an experienced interventional endoscopist, proficient in general interventional techniques (endoscopic mucosal resection, submucosal dissection, endoscopic retrograde cholangiopancreatography (ERCP), and endoscopic suturing using the OverStitch device (e.g. transoral gastric outlet reduction)) and other EBTs (Endobarrier and Primary Obesity Surgery Endoluminal (POSE)). Before performing ESG, he also attended a 2-day dedicated training course followed by four proctored ESG cases.

Patients with obesity were referred for consideration of ESG and were deemed eligible if they were: $\geq 18$ years old, had a $\mathrm{BMI} \geq 30 \mathrm{~kg} / \mathrm{m}^{2}$ and had declined or were not deemed eligible for weight-loss surgery. All patients had already participated in a medical weight-loss management program. A comprehensive surgical, anesthetic, and psychiatric pre-assessment was performed in all cases and informed, written consent was obtained before the procedure. Nutritional assessment and education were delivered by a specialist dietitian. To comply with governance policies, all procedures were performed after necessary approvals were sought and granted by the local Medical Advisory Committee (MAC).

\section{Technique}

Between January 2016 and June 2016, consecutive patients underwent ESG with the previously described triangular configuration of plications, henceforth referred to as pattern (1): "no longitudinal compression”. Thereafter, between June 2016 and 


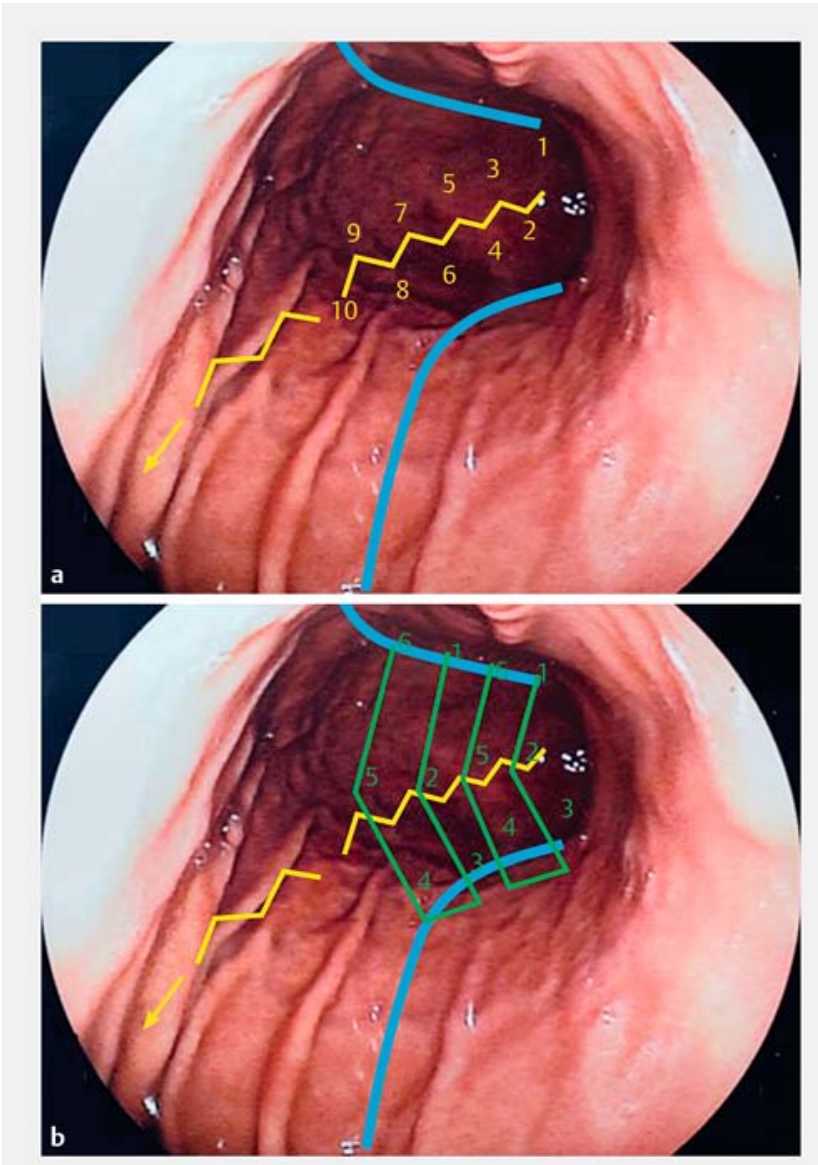

- Fig. 2 Schematic illustration of the modified suture pattern used in our center with (a) initial placement of longitudinal compression sutures, followed by (b) a second parallel layer of "U"-shaped plications.

December 2017, consecutive cases were performed implementing our adapted suture pattern, hereafter referred to as pattern (2): "longitudinal compression".

\section{Suture pattern (1): No longitudinal compression}

This technique has been well described previously [10]. In brief, full thickness bites are taken from the anterior stomach wall, followed by the greater curvature and then the posterior wall before then repeating the pattern in the opposite direction ( Fig.1). Each triangular-shaped plication suture generally consists of 3-9 full-thickness bites that, when clinched together, produce circumferential compression (and, to some degree, shortening) of the greater curvature. Typically, 6-8 plications are used to create the gastric sleeve.

\section{Suture pattern (2): Longitudinal compression}

Using this modified technique, the ESG is started by siting two longitudinal compression sutures along the greater curvature of the stomach; the first suture commences in the proximal antrum and terminates in the mid-body along the greater curve, and the second suture commences in the mid-body and progresses into the fundus along the greater curve ( $\mathbf{F i g . 2 a )}$.
Each linear compression suture consists of approximately 10 bites sited $1.0-1.5 \mathrm{~cm}$ apart so that when each suture is clinched together, the compressive force is distributed evenly along the long-axis of the stomach. The greater curvature is therefore maximally compressed in a concertina-type pattern. Supplementary to this, a second parallel layer of 3-6 interrupted "U"-shaped plications are sited, with each plication being formed of 5-9 bites of the Overstitch ( $\mathbf{F i g} \mathbf{2} \mathbf{2 b}$ ). This second layer acts to produce supplementary compression and volume reduction.

One day before admission, patients were instructed to consume only a clear liquid diet. On the morning of the ESG, a standard oral premedication regime of a proton pump inhibitor (PPI) (Lansoprazole $30 \mathrm{mg}$ ), analgesia (Paracetamol $1 \mathrm{~g}$ ), an anti-inflammatory (Etoricoxib $60 \mathrm{mg}$ ), and an antiemetic (Aprepitant $80 \mathrm{mg}$ ) was prescribed.

After an 8-hour fast, all procedures were carried out in the operating theater under a general anesthetic with the patient in the left-lateral decubitus position with endotracheal intubation. At the time of induction of anesthetic, a prophylactic intravenous dose of Cefuroxime $1.5 \mathrm{~g}$ and Metronidazole $500 \mathrm{mg}$ was administered. An initial gastroscopy was performed using standard $\mathrm{CO}_{2}$ insufflation in order to evaluate the anatomy and ensure that there were no contraindications to performing the ESG, such as gastric ulceration, erosive duodenitis, the presence of a hiatus hernia $>5 \mathrm{~cm}$, or any malignant or premalignant gastric lesions. A full length esophageal overtube was then sited and the OverStitch device, mounted onto a doublechannel endoscope (GIF-2T240; Olympus Medical Systems Corp., Tokyo, Japan), was delivered into the stomach. Starting distally at the level of the incisura angularis and working proximally along the greater curvature to the fundus of the stomach, each plication was formed using a tissue retraction screw (Helix; Apollo Endosurgery Inc., Austin, Texas, United States) to draw the muscularis propria of the stomach wall into the jaws of the Overstitch device in order to take multiple full thickness bites. The bites of each plication were then clinched together to approximate the tissue and produce compressive occlusion of the greater curvature.

Patients were admitted overnight and received regular analgesia, a PPI (Lansoprazole Fast Tabs $30 \mathrm{mg}$ BD), two further doses of intravenous antibiotics, and regular anti-emesis, with strong emphasis placed on avoidance of retching. Patients were permitted to drink water immediately following the procedure and were subsequently progressed to a clear liquid diet for 3 days, a full liquid diet for 2 weeks, pureed food for 2 weeks, and then a soft diet for a further 2 weeks before then returning to ab libitum eating. Patients were advised to consume a low fat, low carbohydrate diet with approximately $60 \mathrm{~g}$ of protein per day and were strongly advised to eat small volumes with cessation of eating immediately at the point of satiety. Dietary follow-up was closely supervised by a specialist dietitian.

\section{Follow-up and outcome measures}

Follow-up appointments with the multidisciplinary team were offered to patients at 1, 3, 6, 9, and 12 months with annual clinical reviews thereafter. During each visit, wellbeing was re- 
- Table1 Baseline patient characteristics and ESG technical data.

\begin{tabular}{|c|c|c|c|}
\hline & No longitudinal compression $(n=9)$ & Longitudinal compression $(n=23)$ & $P$ value \\
\hline Age, mean $\pm S D$, years & $45 \pm 12$ & $43 \pm 10$ & 0.600 \\
\hline Gender, female, n (\%) & $5(56)$ & $18(78)$ & 0.199 \\
\hline Weight, median (range), kg & $113.6(82-156.4)$ & $107(74-136)$ & 0.126 \\
\hline BMI, median (range), $\mathrm{kg} / \mathrm{m}^{2}$ & $35.9(30.9-43.8)$ & $36.5(29.8-42.9)$ & 0.950 \\
\hline Theater time, median (range), min & $135(123-204)$ & $138(90-185)$ & 0.900 \\
\hline Procedural time, median (range), min & $96(85-135)$ & $105(65-139)$ & 0.850 \\
\hline LoS, median (range), days & $1(1-4)$ & $1(1-2)$ & 0.150 \\
\hline
\end{tabular}

viewed by the operating consultant and full dietary counseling was performed by a specialist dietitian. Basic anthropometric measurements were recorded, including weight $(\mathrm{kg})$ and height (m), from which Total Body Weight Loss (TBWL, kg), BMI (= weight $\left.(\mathrm{kg}) /(\text { height }(\mathrm{m}))^{2}\right)$, \%TBWL (Total Body Weight Loss $(\%)=($ TBWL $(\mathrm{kg}) /$ baseline weight $(\mathrm{kg})) \times 100)$, and \%EWL (Excess Weight Loss $(\%)=(T B W L(k g)) /($ baseline weight $(\mathrm{kg})-$ ideal body weight $(\mathrm{kg})) \times 100$ ) were extrapolated. All other clinically relevant qualitative data were also recorded, including the nature and frequency of adverse events and the subjective impact of ESG on portion sizes, appetite, and satiety.

\section{Statistical analysis}

Normally distributed variables are reported as means with standard deviation (SD), and the independent $t$ test was used for comparison of these variables. Non-normally distributed variables are reported as medians with the full range, and the Mann-Whitney $U$ test was used to compare such variables. Categorical variables were compared using the Chi-squared test or Fisher's exact test as appropriate. A $P$ value $<0.05$ was considered to be statistically significant. Data were analyzed using SPSS 24.0 software (IBM Corp., Armonk, New York, United States).

\section{Results}

\section{Patient characteristics}

In total, 32 patients underwent ESG during the study period; the first nine consecutive cases were completed utilizing suture pattern 1 (no longitudinal compression) and the subsequent 23 consecutive cases were completed using suture pattern 2 (longitudinal compression). In the no compression and compression groups, the mean ages were $45 \pm 12$ years and $43 \pm 10$ years, the median baseline weights were $113.6 \mathrm{~kg}$ (range 82.0 156.4 ) and $107 \mathrm{~kg}$ (range $74.0-136.0$ ), and the median baseline BMls were $35.9 \mathrm{~kg} / \mathrm{m}^{2}$ (range $30.9-43.8$ ) and $36.5 \mathrm{~kg} / \mathrm{m}^{2}$ (range 29.8-42.9), respectively. There were five female patients (56\%) in the no longitudinal compression group and 18 $(78 \%)$ in the longitudinal compression group $(P=0.199)$. $>$ Table 1 summarizes the baseline patient characteristics. Procedural and baseline anthropometric data were available for all patients, and follow-up data was available for 6 and 12 patients at 1 month for each group, respectively, 4 and 20 patients at 3 months, and 5 and 7 patients at 6 months. The high attrition rate and loss of data at sequential visits were accounted for by patients not attending review appointments and being lost to follow-up.

\section{Procedural outcomes}

In the no longitudinal compression group, a median of 6 plications (range, 5-8) was used to fashion the endoscopic gastric sleeve, with each plication being created from 4 to 8 full thickness bites. In the second group, two longitudinal compression plications were created in the first instance, each with 10 bites, followed by construction of the second layer of " $U$ "-shaped plications with a median of 4 sutures (range, $3-6$ ), each made up of $5-9$ full-thickness bites. There was no statistically significant difference in procedural time (i.e. time elapsed between commencing and terminating general anesthesia) between the two cohorts with a median procedural time in the no compression group of 96 minutes (range, 85-135 minutes) versus 105 minutes (range, 65-139 minutes) in the longitudinal compression group $(P=0.850)$. All patients were admitted to hospital for observation following their ESG with a median length of stay of 1 day in both groups.

The majority of patients experienced self-limiting symptoms postoperatively, including nausea/vomiting in $71.8 \%$ and epigastric discomfort in $62.5 \%$, which all resolved within 48 hours. These symptoms were reported as mild-to-moderate and were readily controlled with medications. There was no significant difference in postoperative symptoms between the two ESG suture patterns and no serious adverse events were reported in this series.

\section{Weight-loss outcomes}

Weight-loss outcomes at 1, 3, and 6 months for both groups are summarized in $>$ Table 2 and in \$ Fig.3. After 6 months, body weight had decreased by $21.1 \mathrm{~kg}$ (range, $12.2-34.0 \mathrm{~kg}$ ) in the longitudinal compression group versus $10.8 \mathrm{~kg}$ (range, $7.0-25.8 \mathrm{~kg})$ in the no longitudinal compression group $(P=$ 0.042 ). Correspondingly, BMI decreased by $7.8 \mathrm{~kg} / \mathrm{m}^{2}$ (range, $4.9-11.2$ ) and $4.1 \mathrm{~kg} / \mathrm{m}^{2}$ (range, $2.6-7.2$ ) in each group, 
Table 2 Weight loss, BMI, BMI reduction, \%TBWL, and \%EWL at 1, 3, and 6 months following ESG, with and without longitudinal compression sutures.

\begin{tabular}{|c|c|c|c|c|c|c|c|}
\hline & \multicolumn{2}{|l|}{1 month } & \multicolumn{2}{|l|}{3 months } & \multicolumn{2}{|l|}{6 months } & \multirow[t]{2}{*}{$P$ value } \\
\hline & $\begin{array}{l}\text { No longitudinal } \\
\text { compression } \\
(n=6)\end{array}$ & $\begin{array}{l}\text { Longitudinal } \\
\text { compression } \\
(n=12)\end{array}$ & $\begin{array}{l}\text { No longitudi- } \\
\text { nal compres- } \\
\text { sion }(n=4)\end{array}$ & $\begin{array}{l}\text { Longitudinal } \\
\text { compression } \\
(n=20)\end{array}$ & $\begin{array}{l}\text { No longitudinal } \\
\text { compression } \\
(n=5)\end{array}$ & $\begin{array}{l}\text { Longitudinal } \\
\text { compression } \\
(n=7)\end{array}$ & \\
\hline $\begin{array}{l}\text { Weight, median } \\
\text { (range), kg }\end{array}$ & $\begin{array}{l}104.6 \\
(75.0-139.3)\end{array}$ & $\begin{array}{l}98.3 \\
(70.6-130.6)\end{array}$ & $\begin{array}{l}102.2 \\
(95.0-132.0)\end{array}$ & $\begin{array}{l}90 \\
(65.0-120.7)\end{array}$ & $\begin{array}{l}106.6 \\
(71.2-130.6)\end{array}$ & $\begin{array}{l}86.2 \\
(76.8-108.9)\end{array}$ & \\
\hline $\begin{array}{l}\text { BMI, median } \\
\text { (range), } \mathrm{kg} / \mathrm{m}^{2}\end{array}$ & $\begin{array}{l}33.6 \\
(28.2-39.0)\end{array}$ & $\begin{array}{l}33.6 \\
(28.7-40.9)\end{array}$ & $\begin{array}{l}34.7 \\
(28.7-39.4)\end{array}$ & $\begin{array}{l}31.5 \\
(25.7-37.8)\end{array}$ & $\begin{array}{l}33.1 \\
(26.6-39.6)\end{array}$ & $\begin{array}{l}31.8 \\
(23.3-33.3)\end{array}$ & \\
\hline $\begin{array}{l}\text { Weight loss, } \\
\text { median (range), kg }\end{array}$ & $7.8(6.0-17.1)$ & $\begin{array}{l}7.0 \\
(5.4-12.0)\end{array}$ & $\begin{array}{l}10.9 \\
(7.6-24.4)\end{array}$ & $\begin{array}{l}12.2 \\
(9.0-23.0)\end{array}$ & $\begin{array}{l}10.8 \\
(7.0-25.8)^{1}\end{array}$ & $\begin{array}{l}21.1 \\
(12.2-34)^{1}\end{array}$ & $0.042^{1}$ \\
\hline $\begin{array}{l}\text { BMI reduction, me- } \\
\text { dian (range), } \mathrm{kg} / \mathrm{m}^{2}\end{array}$ & $2.5(2.0-4.8)$ & $2.6(1.7-3.7)$ & $3.4(2.8-6.8)$ & $4.7(3.0-7.5)$ & $4.1(2.6-7.2)^{1}$ & $\begin{array}{l}7.8 \\
(4.9-11.2)^{1}\end{array}$ & $0.019^{1}$ \\
\hline \%TBWL, \% (range) & $7.6(5.5-10.9)$ & $\begin{array}{l}6.9 \\
(4.0-10.7)\end{array}$ & $\begin{array}{l}10.1 \\
(6.7-15.6)\end{array}$ & $\begin{array}{l}13.3 \\
(8.6-20.5)\end{array}$ & $\begin{array}{l}13.2 \\
(6.2-17.1)^{1}\end{array}$ & $\begin{array}{l}19.5 \\
(12.9-30.4)^{1}\end{array}$ & $0.042^{1}$ \\
\hline \%EWL, \% (range) & $\begin{array}{l}23.6 \\
(18.0-46.6)\end{array}$ & $\begin{array}{l}22.4 \\
(9.6-44.2)\end{array}$ & $\begin{array}{l}34.1 \\
(16.4-47.9)\end{array}$ & $\begin{array}{l}41 \\
(23.2-86.3)\end{array}$ & $\begin{array}{l}38.5 \\
(15.1-77.8)\end{array}$ & $\begin{array}{l}52.6 \\
(37.2-120.2)\end{array}$ & \\
\hline
\end{tabular}

BMI, body mass index; TBWL, total body weight loss; EWL, excess weight loss; ESG, endoscopic sleeve gastroplasty.

${ }^{1}$ Denotes significant difference between groups.

respectively $(P=0.019)$. In addition, \%TBWL was greater in the longitudinal compression group at $19.5 \%$ (range, $12.9-$ $30.4 \%$ ) compared to $13.2 \%$ (range, $6.2-17.1 \%$ ) in the no longitudinal compression group ( $P=0.042)$. $\mathrm{EWL}$ at 6 months was also greater in the compression group (52.6\% (37.2-120.2\%) vs. $35.5 \%(15.1-77.8 \%)$ but this failed to reach statistical significance $(P=0.291)$.

During follow-up visits, subjective reports on appetite, satiety, and portion sizes were reported for $81 \%(n=26)$ of the patients. Of these, $100 \%$ reported significant early satiety, and portion sizes were reported as being decreased by $50-75 \%$. A significant number of patients also reported an appreciable reduction in their appetite. Two patients in the "no longitudinal compression" group reported an initial good response to their ESG but at 3 months, their weight plateaued and the restrictive effects of the ESG declined. Notably, the first of these patients was hospitalized elsewhere with gastroenteritis and violent vomiting. A follow-up endoscopy demonstrated complete loss of 4 plications, presumed to be a consequence of excessive vomiting. Despite this, the first patient achieved a \%TBWL of $9.0 \%$ and the second achieved $13.2 \%$.

\section{Discussion}

Our experience further supports ESG as a safe and effective means of achieving significant weight loss in obese individuals. Alqahani et al. (2018) have reported on the largest prospective series of ESG patients $(n=1000)$ to date with a follow-up of 18 months [27]. That group used the same suture pattern as the "no longitudinal compression" group in our study, and reported a $\%$ TBWL of $13.7 \pm 6.8 \%, 15.0 \pm 7.7 \%$, and $14.8 \pm 8.5 \%$, and $\%$ EWL of $64.3 \pm 56.2 \%, 67.5 \pm 52.3 \%$, and $64.7 \pm 55.4 \%$ at 6,12 , and 18 months, respectively. At 6 months, the median \%TBWL in our comparative group was $13.2 \%$ (range, 6.2-17.1) and thus comparable to that series. In contrast, \%TBWL achieved by patients undergoing ESG using our unique suture pattern was $19.5 \%$ (range, $12.9-30.4$ ) with a median \%EWL of $52.6 \%$ (range, $37.2-120.2$ ). This is significantly greater than the no compression group within our own series $(P=0.042)$ and also superior to the 6-month \%TBWL outcomes reported by Alqahani et al. Our 6-month \%TBWL outcomes in this group are also superior to the 6-month outcomes and comparable to the 12 and 24-month outcomes reported in a prospective series by Lopez-Nava et al. [26], which currently represents the best weight-loss outcomes reported in any study of ESG to date. We await our longer-term follow-up data for direct comparison. However, we postulate that the longitudinal compression sutures create superior "concertina-like" compression of the greater curvature, further reducing the volume of the gastroplasty and therefore potentially further supporting the remaining sutures. As a result, satiety increases and portion sizes reduce, as evidenced by our qualitative data.

Adopting this new suture pattern resulted in no additional morbidity or mortality and can be considered as safe practice. Additionally, fashioning of these longitudinal compression sutures is technically straightforward and does not result in any significant increase in procedural time. The transient post-procedural symptoms of nausea and abdominal discomfort are in line with other reported studies and there was no demonstrable difference in symptoms between suture patterns. Unlike other studies that have reported cases of peri-gastric collections, upper gastrointestinal bleeds and perforation, we are yet to report any serious adverse events following ESG.

We recognize important limitations in this study including its retrospective nature, the short duration of follow-up data available, and the small number of patients included. Addition- 

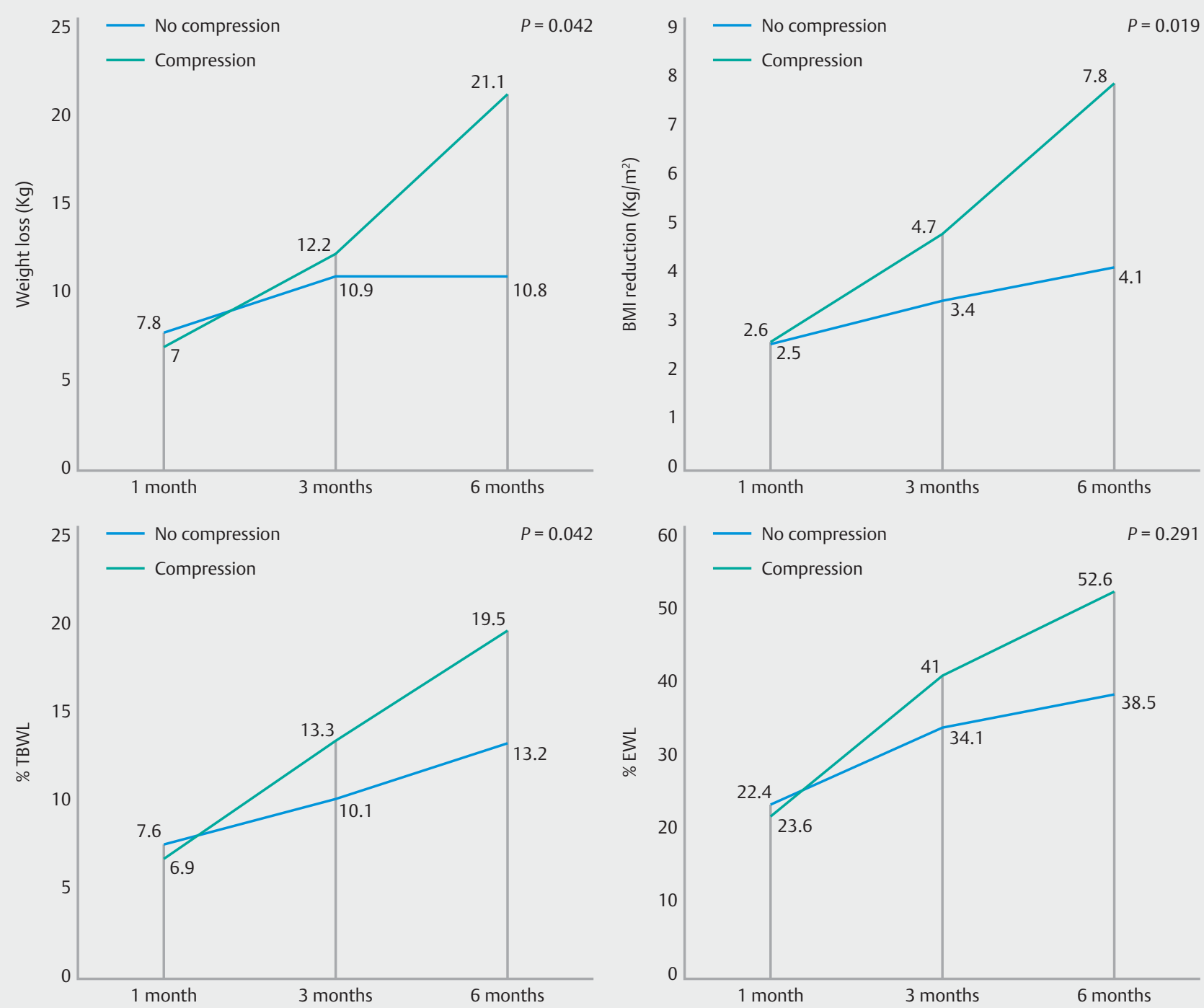

Fig. 3 Weight loss ( $\mathrm{kg})$, BMI reduction $\left(\mathrm{kg} / \mathrm{m}^{2}\right), \%$ TBWL, and \%EWL at 1, 3, and 6 months following endoscopic sleeve gastroplasty (ESG) with and without longitudinal compression sutures. BMI, body mass index; TBWL, total body weight loss; EWL, excess weight loss.

ally, all procedures were conducted by a single surgeon, which limits the generalizability of our findings, and the operator learning curve of this procedure may be considered as a potential confounding factor. Saumoy et al. (2017) [14] reviewed the learning curve of a single operator performing ESG and concluded that efficiency was attained after performing 38 cases, with mastery after 55 cases. They also concluded that there were no significant differences in weight-loss outcomes before and after reaching the status of efficiency. It is therefore unlikely that the operator learning curve of the 32 patients reported in this study contributed to the differences in weight loss observed between the two suture patterns.

Nonetheless, our outcomes give preliminary evidence that additional longitudinal compression plications along the greater curvature of the stomach may result in superior weight loss and, therefore, further research into this finding in the form of a prospective randomized controlled trial is justified. Using our 6- month follow-up data, using \%TBWL as the primary outcome measure, a sample size of $n=29$ (90\% power with a 0.05 significance level and accounting for $20 \%$ loss to follow-up) would be required.

In conclusion, ESG is now established as a safe and efficacious minimally invasive treatment modality in the armamentarium against obesity and obesity-related diseases. The technique of ESG is evolving and outcomes from EBTs continue to improve. We provide preliminary evidence of superior weight loss achieved through a modified gastroplasty suture pattern but further prospective data are required to validate these findings. 


\section{Acknowledgments}

Special thanks are given to Apollo Healthcare Technologies, Spire Healthcare Southampton, Dr. Gontrand Lopez-Nava, and Healthier Weight for their ongoing support of this work.

\section{Competing interests}

MAG has no conflicts of interest or financial ties to disclose. ALM has no conflicts of interest or financial ties to disclose. $J \mathrm{~K}$ is a proctor for Apollo Endosurgery in the UK.

\section{References}

[1] World Health Organization. Obesity and overweight fact sheet: World Health Organization. Updated 18th October 2017. 2017: Available from: http://www.who.int/en/news-room/fact-sheets/detail/obesityand-overweight [Accessed 1 June 2018]

[2] Schauer PR, Bhatt DL, Kirwan JP et al. Bariatric surgery versus intensive medical therapy for diabetes - 5-year outcomes. NEJM 2017; 376: $641-651$

[3] Buchwald H, Estok R, Fahrbach $\mathrm{K}$ et al. Weight and type 2 diabetes after bariatric surgery: systematic review and meta-analysis. Am J Med 2009; 122: 248-256 e5

[4] Sjöström L. Review of the key results from the Swedish Obese Subjects (SOS) trial - a prospective controlled intervention study of bariatric surgery. J Intern Med 2013; 273: 219-234

[5] Rubino F, Nathan D, Eckel R et al. Metabolic surgery in the treatment algorithm for type 2 diabetes: a joint statement by international diabetes organizations. Diabetes Care 2016; 39: 861 - 877

[6] Aminian A, Brethauer SA, Kirwan JP et al. How safe is metabolic/diabetes surgery? Diabetes Obes Metab 2015; 17: 198-201

[7] Afonso BB, Rosenthal R, Li KM et al. Perceived barriers to bariatric surgery among morbidly obese patients. Surg Obes Relat Dis 2010; 6 : $16-21$

[8] Fogel R, De Fogel J, Bonilla Y et al. Clinical experience of transoral suturing for an endoluminal vertical gastroplasty: 1 -year follow-up in 64 patients. Gastrointest Endosc 2008; 68: $51-58$

[9] Abu Dayyeh BK, Acosta A, Camilleri M et al. Endoscopic sleeve gastroplasty alters gastric physiology and induces loss of body weight in obese individuals. Clin Gastroenterol Hepatol 2017; 15: 37 - 43 e1

[10] Lopez-Nava G, Galvao MP, Bautista-Castano I et al. Endoscopic sleeve gastroplasty: how I do it? Obes Surg 2015; 25: 1534 - 1538

[11] Sartoretto A, Sui Z, Hill C et al. Endoscopic sleeve gastroplasty (ESG) is a reproducible and effective endoscopic bariatric therapy suitable for widespread clinical adoption: a large, international multicenter study. Obes Surg 2018; 28: $1812-1821$
[12] Graus Morales ], Crespo Perez L, Marques A et al. Modified endoscopic gastroplasty for the treatment of obesity. Surg Endosc 2018; 32: $3936-3942$

[13] Sharaiha RZ, Kumta NA, Saumoy M et al. Endoscopic sleeve gastroplasty significantly reduces body mass index and metabolic complications in obese patients. Clin Gastroenterol Hepatol 2017; 15: $504-$ 510

[14] Saumoy M, Schneider Y, Zhou XK et al. A single-operator learning curve analysis for the endoscopic sleeve gastroplasty. Gastrointest Endosc 2017; 87: 442-447

[15] Novikov AA, Afaneh C, Saumoy M et al. Endoscopic sleeve gastroplasty, laparoscopic sleeve gastrectomy, and laparoscopic band for weight loss: how do they compare? J Gastrointest Surg 2017; 22: $267-273$

[16] Lopez-Nava G, Sharaiha RZ, Vargas E] et al. Endoscopic sleeve gastroplasty for obesity: a multicenter study of 248 patients with 24 months follow-up. Obes Surg 2017; 27: 2649-2655

[17] Kumar N, Abu Dayyeh BK, Lopez-Nava Breviere G et al. Endoscopic sutured gastroplasty: procedure evolution from first-in-man cases through current technique. Surg Endosc 2017; 32: 2159-2164

[18] Hill C, El Zein M, Agnihotri A et al. Endoscopic sleeve gastroplasty: the learning curve. Endosc Int Open 2017; 5: E900 - E904

[19] Eid G. Sleeve gastrectomy revision by endoluminal sleeve plication gastroplasty: a small pilot case series. Surg Endosc 2017; 31: 4252 4255

[20] Lopez-Nava G, Galvao M, Bautista-Castano I et al. Endoscopic sleeve gastroplasty with 1-year follow-up: factors predictive of success. Endosc Int Open 2016; 4: E222-E227

[21] Galvao-Neto MD, Grecco E, Souza TF et al. Endoscopic sleeve gastroplasty - minimally invasive therapy for primary obesity treatment. Arq Bras Cir Dig 2016; 29: 95 - 97

[22] Sharaiha RZ, Kedia P, Kumta N et al. Initial experience with endoscopic sleeve gastroplasty: technical success and reproducibility in the bariatric population. Endoscopy 2015; 47: $164-166$

[23] Lopez-Nava G, Galvao MP, da Bautista-Castano I et al. Endoscopic sleeve gastroplasty for the treatment of obesity. Endoscopy 2015; 47 : $449-452$

[24] Kumar N, Sahdala HNP, Shaikh S et al. Mo1155 endoscopic sleeve gastroplasty for primary therapy of obesity: initial human cases. Gastroenterology 2014; 146: S571 - S572

[25] Abu Dayyeh BK, Rajan E, Gostout C]. Endoscopic sleeve gastroplasty: a potential endoscopic alternative to surgical sleeve gastrectomy for treatment of obesity. Gastrointest Endosc 2013; 78: 530-535

[26] Lopez-Nava G, Galvao M, Bautista-Castano I et al. Endoscopic sleeve gastroplasty for obesity treatment: two years of experience. Arq Bras Cir Dig 2017; 30: 18 - 20

[27] Alqahtani A, Al-Darwish A, Mahmoud AE et al. Short-term outcomes of endoscopic sleeve gastroplasty in 1000 consecutive patients. Gastrointest Endosc 2019; 89: 1132-1138 